Check for updates

Cite this: Chem. Sci., 2019, 10, 9257

๑ All publication charges for this article have been paid for by the Royal Society of Chemistry

Received 6th June 2019

Accepted 16th August 2019

DOI: $10.1039 / c 9 s c 02764 \mathrm{e}$

rsc.li/chemical-science

\section{Engineering an NIR rhodol derivative with spirocyclic ring-opening activation for high- contrast photoacoustic imaging $\dagger$}

\author{
Feng Liu, Xiao Shi, Xianjun Liu, (D) Fenglin Wang, (DD * Hai-Bo Yi (D) \\ and Jian-Hui Jiang (D) *
}

Molecular probes that enable high-contrast photoacoustic (PA) imaging of cellular processes are valuable tools for in vivo studies. Design of activatable PA probes with high contrast remains elusive. We develop a new NIR rhodol derivative, Rhodol-NIR, with a large extinction coefficient, low quantum yield and structural switching from a 'ring-open' form to a 'closed' spirolactone upon esterification. This structural transition, together with the ideal photophysical properties, enables the development of activatable probes for high-contrast PA imaging via a target-specific de-esterification reaction. This strategy is demonstrated using a PA probe designed for a tumor biomarker, human NAD(P)H: quinone oxidoreductase isozyme 1 (hNQO1), which affords high contrast and excellent sensitivity for PA detection and imaging of $\mathrm{hNQO1}$ in living cells and animals. The strategy can provide a new paradigm for engineering activatable PA probes for high-contrast imaging.

\section{Introduction}

Photoacoustic (PA) imaging is emerging as a powerful biomedical imaging modality that enables non-invasive visualization of biological processes at molecular and cellular levels in deep tissues with high spatial resolution..$^{1-3}$ Using the nearinfrared (NIR) operating window, PA imaging achieves a penetration depth of a few centimeters with a resolution of $\sim 100$ $\mu \mathrm{m} .{ }^{4,5}$ Because of these advantages, it is a useful tool for preclinical in vivo imaging of various diseases and for diagnosis, metastatic assessment and therapeutic monitoring of cancer.

Molecular probes are essential in PA imaging because they can impart molecular or cellular specificity and enhance imaging contrast. ${ }^{6}$ PA probes have typically relied on designs that allow selective accumulation of photon-absorbing agents in the diseased areas via either passive or active targeting strategies. $^{7-9}$ Activatable PA probes, which can cause PA signal enhancement upon interaction with a specific molecular or cellular event, are particularly promising. These probes confer advantages of low background and high sensitivity, facilitating real-time imaging with higher depth and spatial resolution..$^{10}$ Current activatable PA probes have been mainly designed based on the intramolecular charge transfer (ICT) ${ }^{11-16}$ and the contact quenching mechanisms. ${ }^{17,18}$ These two mechanisms have been

State Key Laboratory of Chemo/Biosensing \& Chemometrics, College of Chemistry \& Chemical Engineering, Hunan University, Changsha 410082, China. E-mail: jianhuijiang@hnu.edu.cn; fengliw@hnu.edu.cn

$\uparrow$ Electronic supplementary information (ESI) available: Procedures for synthesis, characterization data, and supplementary figures. See DOI: 10.1039/c9sc02764e well demonstrated for activatable PA probes with applications explored for several molecular targets, such as metal ions, ${ }^{11,12}$ reactive oxygen species, ${ }^{13}$ nitric oxide, ${ }^{14}$ hypoxia, ${ }^{15}$ and enzymes. ${ }^{16-18}$ However, most of these probes still suffer from limited imaging contrast due to small changes in absorption spectral shifts or quantum yields. Rational design of activatable PA probes with ideal photophysical properties, such as a large absorption spectral shift, intense NIR absorption, low quantum yield and high photostability, which ultimately deliver highcontrast PA signals, remains elusive.

Spirocyclic xanthene dyes, such as fluorescein and rhodol, are known to be present in equilibrium between a 'ringopening' form and a 'closed' spirocyclic structure with distinct $\pi$-conjugation systems. ${ }^{19-22}$ The 'ring-opening' form exhibits a red-shifted and enhanced absorption band as compared to the 'closed' structure, holding great potential for designing highcontrast PA probes. There have been a few studies using spirocyclic xanthene dyes for PA imaging, including a visible light absorbing dye, seminaphthorhodafluor-5F, for $\mathrm{pH}$ imaging, ${ }^{23,24}$ and an NIR absorbing dye, silicon-rhodamine, for hypochlorous acid imaging. ${ }^{25}$ These studies all involve direct ring-opening reactions between the spirocyclic moiety and the analyte. Such ring-opening transitions, to our knowledge, are still very limited. ${ }^{26}$ New NIR absorbing spirocyclic xanthene dyes with generally applicable ring-opening responsiveness have not been explored.

Herein we report a new spirocyclic xanthene dye with intense NIR absorption, low quantum yield and generally applicable ring-opening responsiveness for developing a high-contrast PA probe, as illustrated in Scheme 1. The spirocyclic xanthene dye, 
(a)
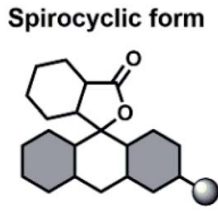

PA "off"

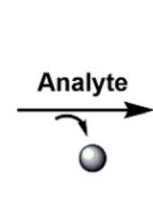

(b)

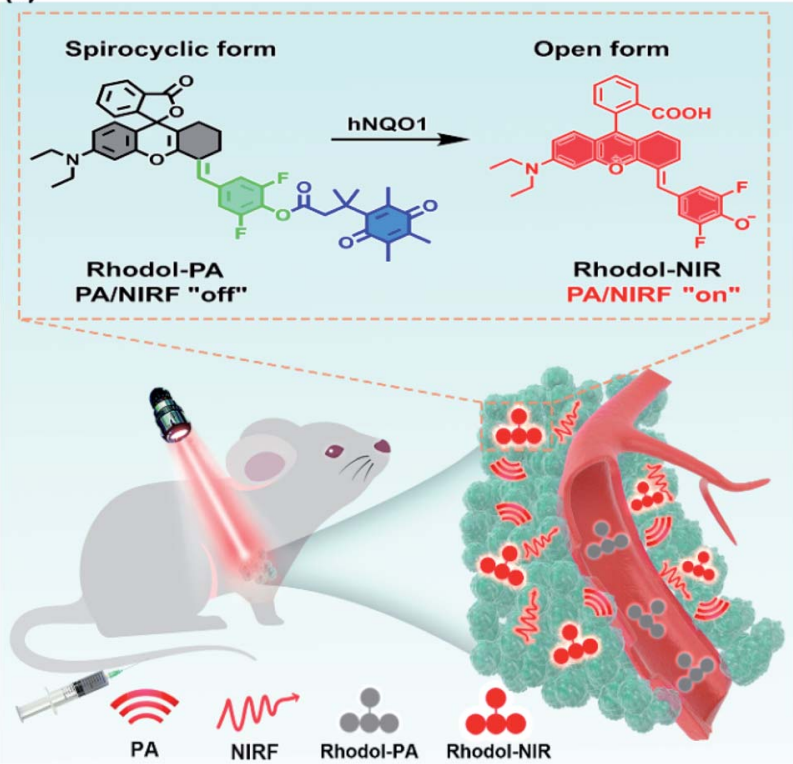

Scheme 1 (a) Schematic illustration of the analyte-mediated spirocyclic ring opening reaction for activatable PA imaging. (b) Illustration of the mechanism of Rhodol-PA for PA/NIRF imaging.

when esterified at the phenolic hydroxyl group, has a dominant spirocyclic structure. This spirocyclic form has a disrupted conjugation system in the xanthene core, thereby displaying negligible absorption in the NIR region (Scheme 1a). The analyte uncages the phenolic hydroxyl moiety with a concomitant 'ring-opening' transition, which restores the conjugated xanthene structure, conferring strong NIR absorption with a high-contrast PA signal. Based on this design, we engineer a new rhodol derivative by conjugating the core rhodol structure with a difluorophenol moiety (Scheme 1b). This new rhodol derivative, Rhodol-NIR, has an extended $\pi$-conjugation system and a finely-tuned protolytic constant for the phenolic hydroxyl moiety, exhibiting excellent photophysical properties for highcontrast PA imaging. Moreover, it forms a closed spirolactone structure when the phenolic hydroxyl moiety is esterified, while it recovers the 'ring-open' form upon de-esterification. Moreover, there are diverse reactions to uncage the phenolic hydroxyl moiety in response to varying the analyte. Therefore, this new Rhodol-NIR dye affords a useful platform for developing a highcontrast PA probe with generally applicable ring-opening responsiveness.

To demonstrate its utility, we chose human $\mathrm{NAD}(\mathrm{P}) \mathrm{H}$ : quinone oxidoreductase isozyme 1 (hNQO1), a known biomarker enzyme commonly overexpressed in tumor cells, ${ }^{27-29}$ as the case of study. The PA probe for hNQO1 is designed by cloaking the hydroxyl group with a trimethyl locked quinone propionic acid moiety $\left(\mathrm{Q}_{3}\right)$. The PA probe displays only negligible absorption in the NIR region. After hNQO1-catalyzed elimination of the $\mathrm{Q}_{3}$ moiety, the PA probe is converted into the Rhodol-NIR dye with a spontaneous ring-opening process, generating a high signal-to-background PA response. In addition, the Rhodol-NIR dye also delivers a fluorescence signal, permitting dual-mode detection of the enzyme using PA and NIR fluorescence (NIRF) signals. The PA probe is demonstrated to allow high-contrast PA/NIRF dual-mode imaging and ascertaining of tumors with differential hNQO1 expression in living cells and animals, affording great diagnostic potential for hNQO1-overexpression associated diseases. To our knowledge, this is the first time that a Rhodol probe has been developed with NIR absorption, low quantum yield and generally applicable responsiveness.

\section{Results and discussion}

\section{Design and synthesis of Rhodol-NIR}

We chose rhodol as the core structure to engineer a new spirocyclic xanthene chromophore due to its excellent photophysical properties. ${ }^{30,31}$ First, a rhodol variant, Rhodol-NIR1 (Fig. 1a), was synthesized by conjugating the core rhodol structure with 4-hydroxybenzaldehyde via the classic Knoevenagel reaction..$^{32}$ This reaction not only allowed facile synthesis, but also rendered a non-rigid structure with low quantum yield. Surprisingly, Rhodol-NIR1 only showed a slight red shift in the absorption peak $(570 \mathrm{~nm})$ versus rhodol $(550 \mathrm{~nm})$ at $\mathrm{pH} 7.4$ except for a relatively large shift in the fluorescence peak (Fig. 1b and $\mathrm{S} 1 \dagger$ ). A closer examination revealed that Rhodol-NIR1 displayed bathochromic shifts at higher $\mathrm{pH}$, and the absorption maximum was $\sim 650 \mathrm{~nm}$ at $\mathrm{pH} 10.0$. The fluorescence maximum

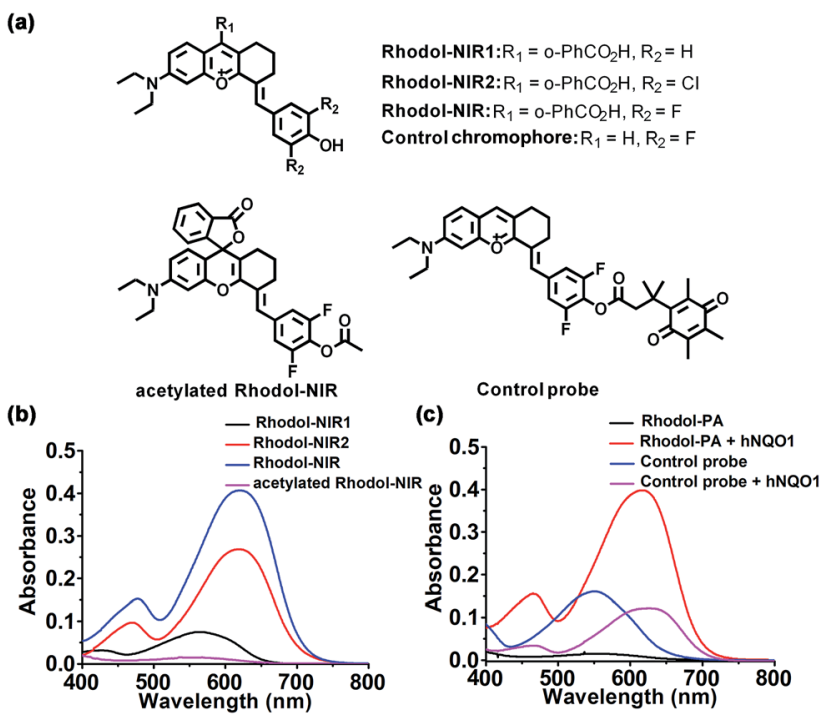

Fig. 1 (a) Chemical structures of Rhodol-NIR, Rhodol-NIR1, RhodolNIR2, the control chromophore, acetylated Rhodol-NIR and the control probe; (b) UV-Vis absorption spectra of Rhodol-NIR, RhodolNIR1, Rhodol-NIR2 and acetylated Rhodol-NIR (15 $\mu \mathrm{M})$ at $\mathrm{pH} 7.4$; (c) UV-Vis absorption responses of Rhodol-PA and the control probe (15 $\mu \mathrm{M})$ towards hNQO1 $\left(3.0 \mu \mathrm{g} \mathrm{mL}^{-1}\right)$. 
was also shifted from $660 \mathrm{~nm}$ to $720 \mathrm{~nm}$ as the $\mathrm{pH}$ increased from 7.4 to 10.0 (Fig. S2 $\dagger$ ). The pH-dependent absorption of Rhodol-NIR1 was ascribed to the transition from the acidic form to the basic one due to prototropic dissociation of the phenolic hydroxyl group at higher $\mathrm{pH}$. The $\mathrm{p} K_{\mathrm{a}}$ was determined to be $\sim 8.7$, indicating that Rhodol-NIR1 was present mainly in the acidic form under physiological conditions. Motivated by the observation that the basic form exhibited NIR absorption, we then attempted to decrease the $\mathrm{p} K_{\mathrm{a}}$ of the phenolic hydroxyl moiety by introducing electron-withdrawing substituents at its ortho positions (Fig. 1a). As anticipated, a derivative of RhodolNIR1 with difluoro-substitution, Rhodol-NIR, exhibited an enhanced absorption band in the NIR region from $620 \mathrm{~nm}$ to $700 \mathrm{~nm}$ with a fluorescence peak at $720 \mathrm{~nm}$ at pH 7.4 (Fig. S1 $\dagger$ ). The molar extinction coefficients at $630 \mathrm{~nm}$ and $680 \mathrm{~nm}$ were $2.67 \times 10^{5} \mathrm{M}^{-1} \mathrm{~cm}^{-1}$ and $1.13 \times 10^{5} \mathrm{M}^{-1} \mathrm{~cm}^{-1}$, respectively (Fig. $1 \mathrm{~b}$ and Table $\mathrm{S} 1 \dagger$ ). The quantum yield $\Phi_{\mathrm{F}}$ was $1.0 \%$ at $\mathrm{pH}$ 7.4 in an aqueous solution. This low quantum yield indicated that Rhodol-NIR exhibited high efficiency in photo-to-thermal conversion for PA imaging, considering it had no substituents of heavy atoms associated with a competitive photo conversion pathway. ${ }^{33}$ Moreover, the $\mathrm{p} K_{\mathrm{a}}$ of Rhodol-NIR was estimated to be 6.1, indicating a dominant contribution of its basic form to the NIR absorption at pH 7.4 (Fig. S3†). A similar derivative with dichlorine-substitution, Rhodol-NIR2, also gave a substantial NIR absorption peak at $\mathrm{pH} 7.4$ (Fig. $1 \mathrm{~b}$ and $\mathrm{S} 4 \dagger$ ). This result suggested that electron-withdrawing substituents were beneficial for the decrease of $\mathrm{p} K_{\mathrm{a}}$ and enhancement of NIR absorption of rhodol derivatives at $\mathrm{pH}$ 7.4. Because Rhodol-NIR has stronger vis-NIR absorption at $\mathrm{pH}$ 7.4, it was chosen for further studies. In addition, Rhodol-NIR was found to exhibit superior photostability as compared to a commercial NIR dye, indocyanine green (ICG) (Fig. S5 $\dagger$ ). The high photostability also suggested its utility for PA imaging.

\section{Engineering of the Rhodol-NIR based PA probe for hNQO1}

To test the potential of Rhodol-NIR for developing highcontrast PA probes, acetylated Rhodol-NIR was first synthesized (Fig. 1a). As anticipated, acetylated Rhodol-NIR did not show an absorption band in the vis-NIR region, implying that it was present in the 'closed' spirolactone structure with a disrupted $\pi$-conjugation system (Fig. 1b). This finding revealed that Rhodol-NIR has the potential for developing high-contrast activatable PA probes. We also attempted to further extend the conjugation system by conjugating the rhodol core with other coumarin or xanthene structures, respectively. Unfortunately, their acetylated counterparts both failed to exhibit substantial contrast in the NIR region as compared to the corresponding dye (Fig. S6 $†$ ), indicating that these further engineered dyes could not exhibit the desired spirocyclic ring-opening activation property. Because RhodolNIR exhibits strong absorption in the NIR region (650-700 $\mathrm{nm}$ ), we used it to demonstrate the capability of developing an activatable PA probe for hNQO1.

To develop an activatable probe for PA imaging of hNQO1, the probe, Rhodol-PA, was synthesized by esterification of
Rhodol-NIR at the phenolic hydroxyl group with $\mathrm{Q}_{3}$ (Scheme 1b). The resulting Rhodol-PA probe did not show an absorption band in the vis-NIR region (Fig. 1c). After 90 min incubation with hNQO1, we observed a distinct NIR absorption band at $630 \mathrm{~nm}$, indicating the production of Rhodol-NIR (Fig. 1c). This finding manifested a two-step mechanism for the hNQO1 catalyzed reaction, in which reduction-elimination of the cloaking moiety $\mathrm{Q}_{3}$ in Rhodol-PA was spontaneously followed by the spirocyclic ring opening transition. Note that the Rhodol-PA probe exhibited very desirable response performance to hNQO1 with a large bathochromic shift $(\Delta \lambda>230 \mathrm{~nm})$ and high signalto-background ratio ( $>57$-fold at $630 \mathrm{~nm}$ and $>110$-fold at 680 $\mathrm{nm})$. This ideal performance was attributed to the generally applicable ring-opening responsiveness, in which the probe exhibited the 'closed' spirolactone structure with a disrupted $\pi$-conjugation while the product had the 'ring-open' form with substantial $\pi$-conjugation extension.

To validate the advantage of the spirocyclic ring-opening design, we synthesized a control chromophore using a variant of Rhodol-NIR without the spirocyclic moiety (Fig. 1a). A control probe was prepared by esterifying the chromophore with $\mathrm{Q}_{3}$ (Fig. 1a). Interestingly, the control probe did exhibit an obvious vis-NIR absorption band (Fig. 1c). It was noteworthy that the control probe shared the same core structure as the Rhodol-PA probe. Hence, the distinct absorption spectra for the control probe and Rhodol-PA gave direct evidence for a disrupted $\pi$ conjugation system in Rhodol-PA, verifying the 'closed' spirolactone structure in Rhodol-PA. Moreover, after the control probe was incubated with hNQO1, a slight increase appeared in the absorption peak with only 3-fold enhancement of the maximum. This slight absorption increase was ascribed to an enhanced intramolecular charge transfer effect owing to the formation of a free phenolic hydroxyl group after the enzymatic reaction. Based on these results, we reasoned that the highcontrast response of Rhodol-PA was attributed to the generally applicable ring-opening activation, validating the potential of this new strategy for developing high-contrast activatable PA probes.

A closer theoretical examination was performed to gain insight into the optical properties of the engineered chromophores and probes. TDDFT (time-dependent density functional theory) calculations revealed that substitution with fluorine at the ortho positions of phenolic hydroxyl narrowed the energy gaps between the highest occupied molecular orbital (HOMO) and lowest unoccupied molecular orbital (LUMO) of RhodolNIR. The decreased HOMO-LUMO energy gaps accounted for the red-shifted absorption of Rhodol-NIR as compared to Rhodol-NIR1 (Table S2 and Fig. S7 $\dagger$ ). Acetylated Rhodol-NIR, a simplified variant of Rhodol-PA, was calculated to have a much larger HOMO-LUMO energy gap than Rhodol-NIR, indicating a largely hypochromatic shift for the absorption band of Rhodol-PA. Moreover, Rhodol-NIR had decreased electronegativity for the oxygen atom of the phenolic hydroxyl moiety as compared to Rhodol-NIR1, which might explain the smallest $\mathrm{p} K_{\mathrm{a}}$ for Rhodol-NIR. Additionally, the acetylated control chromophore, a variant of Rhodol-NIR without the spirocyclic moiety, had a much smaller HOMO-LUMO energy 
gap, which may account for its substantial absorption in the visNIR region. These theoretical calculations were consistent with our experimental data.

The reaction mechanism of the Rhodol-PA probe was further verified using reversed phase high performance liquid chromatography (RP-HPLC) and high-resolution electrospray ionization (HR-ESI-MS). Two elution peaks were obtained for the products from the reaction between the probe and hNQO1 at 8.8 and $5.0 \mathrm{~min}$, respectively, corresponding to the Rhodol-NIR chromophore and the unreacted probe (Fig. S8 $\dagger$ ). Moreover, the product showed two new peaks, one corresponding to Rhodol-NIR (calculated for $[\mathrm{M}+\mathrm{H}]^{+} \mathrm{m} / \mathrm{z}$ 516.1981, found 516.1987) and the other for the reduction by-product (calculated for $[\mathrm{M}+\mathrm{H}]^{+} m / z$ 235.1289, found 235.0541) (Fig. S9 $\dagger$ ). This result implied that the Rhodol-PA probe was converted to Rhodol-NIR upon reaction with hNQO1.

\section{In vitro performance of the PA probe for hNQO1}

Having obtained the desired Rhodol-PA probe, we then investigated its performance as a PA probe for in vitro hNQO1 detection. As expected, the Rhodol-PA probe exhibited very low PA signals from $680 \mathrm{~nm}$ to $800 \mathrm{~nm}$ (Fig. 2a). After the probe was incubated with hNQO1, intense PA responses were obtained with a high signal-to-background ratio of $\sim 11$-fold at $680 \mathrm{~nm}$.
Similarly, the Rhodol-PA probe only exhibited a very low fluorescence, while its reaction with hNQO1 generated a substantial enhancement in fluorescence with a high signal-to-background ratio of $\sim 59$-fold at $720 \mathrm{~nm}$ (Fig. $2 \mathrm{~b}$ and $\mathrm{S} 10 \dagger$ ). This result implied the potential of Rhodol-PA for high-contrast PA/NIRF dual-mode imaging of hNQO1. To test its specificity, control experiments were performed in which the probe was incubated with various biologically relevant species. No appreciable PA, fluorescence and absorption responses were obtained in these assays (Fig. 2c and S11 $\dagger$ ), demonstrating the high selectivity of the Rhodol-PA probe for hNQO1. Further evidence was obtained from a lysate assay of HT-29 cells, which are known for overexpression of hNQO1. ${ }^{27}$ After incubation of the probe with the HT-29 cell lysate, we obtained an appreciable PA signal with strong absorption and fluorescence responses (Fig. 2c and $\mathrm{S} 12 \dagger)$. In contrast, negligible PA, absorption and fluorescence signals were observed in the cell lysates pretreated with excessive dicoumarol, a specific hNQO1 inhibitor. Additionally, incubation the probe with lysates of MDA-MB-231 cells, with extremely low expression of hNQO $1,{ }^{28}$ also showed a very small response in PA, absorption and fluorescence measurements (Fig. 2c and S13†). Taken together, these results clearly demonstrated the high specificity of the Rhodol-PA probe towards hNQO1 activity.
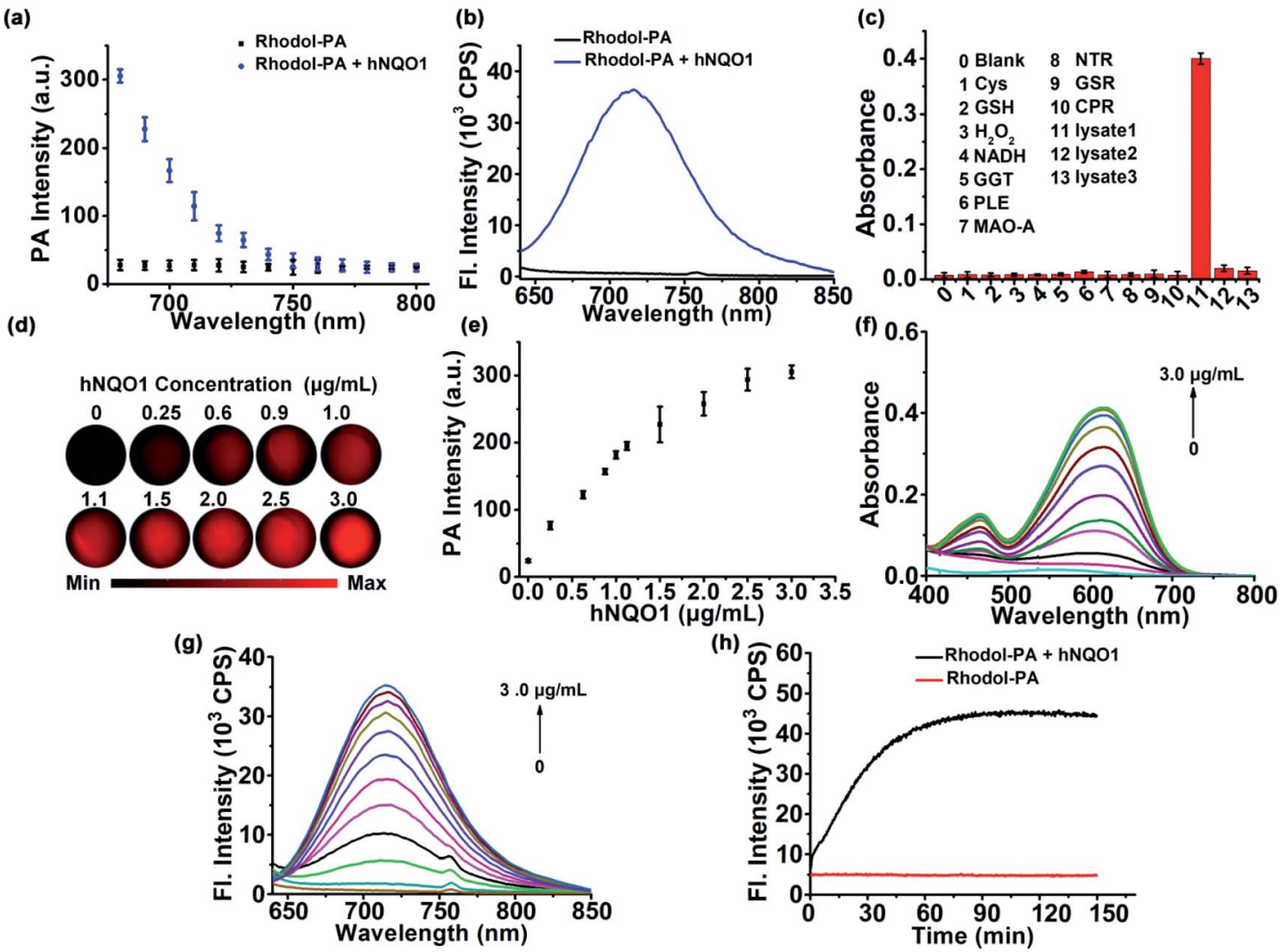

Fig. 2 In vitro characterization of Rhodol-PA towards hNQO1. Responses of Rhodol-PA (15 $\mu \mathrm{M})$ towards hNQO1 (3.0 $\mu \mathrm{g} \mathrm{mL} \mathrm{mL}^{-1}$ ) based on PA (a) and fluorescence spectra (b). (c) Selectivity of Rhodol-PA $(15 \mu \mathrm{M})$ towards various relevant substances based on absorption. Lysate 1 was from HT-29 cells, lysate 2 was from HT-29 cells with an inhibitor, and lysate 3 was from MDA-MB-231 cells. PA (d and e), absorbance (f), and fluorescence $(\mathrm{g})$ responses of Rhodol-PA $(15 \mu \mathrm{M})$ toward various concentrations of hNQO1 $\left(0-3.0 \mu \mathrm{g} \mathrm{mL} \mathrm{m}^{-1}\right.$ ). (h) Real-time fluorescence of RhodolPA $(15 \mu \mathrm{M})$ with or without addition of hNQO1 $\left(3.0 \mu \mathrm{g} \mathrm{mL}{ }^{-1}\right)$. Ex/Em: $620 \mathrm{~nm} / 720 \mathrm{~nm}$. 
Next, we investigated the quantitative ability of the RhodolPA probe. The PA signals at $680 \mathrm{~nm}$ were found to exhibit dynamic responses toward hNQO1 in the concentration range of $0.25-3.0 \mu \mathrm{g} \mathrm{mL} \mathrm{m}^{-1}$ (Fig. 2d and e). The signal-to-background ratio was $\sim 11$, indicating a high contrast in PA detection of hNQO1. A linear correlation was obtained in the range from 0.25 to $1.1 \mu \mathrm{g} \mathrm{mL}^{-1}$ with an estimated limit of detection (LOD) of $0.08 \mu \mathrm{g} \mathrm{mL} \mathrm{m}^{-1}$ (Fig. S13†). Dynamic responses were also obtained with absorption and fluorescence analyses (Fig. $2 \mathrm{f}$ and $\mathrm{g}$ and $\mathbf{S 1 3}$ ). These results confirmed the capability of the Rhodol-PA probe for quantifying hNQO1 concentration. In addition, real-time fluorescence monitoring of the reaction between the probe and hNQO1 demonstrated fast kinetics and the reaction was completed within $30 \mathrm{~min}$ (Fig. 2h). A closer investigation revealed that the Michaelis constant $K_{\mathrm{m}}$ was estimated to be $14.1 \mu \mathrm{M}$ and the catalytic rate constant $k_{\text {cat }}$ was $0.11 \mathrm{~s}^{-1}$ (Fig. S14†), which were comparable to the literature reported values. ${ }^{27-29}$ Additionally, the fluorescence signals of the Rhodol-PA probe was found to remain very low and showed little change over the $\mathrm{pH}$ range from $\mathrm{pH} 4.0$ to 9.0, indicating its stability in the whole physiological $\mathrm{pH}$ range. The probe also exhibited a maximum fluorescence signal under weakly acidic and neutral conditions, implying that the probe gave the optimal response to detect cytosolic enzymes in cancer cells (Fig. S15†).

\section{PA/NIRF imaging of hNQO1 in living cells}

Motivated by the desirable in vitro results, we then explored the capability of the Rhodol-PA probe for PA/NIRF imaging of hNQO1 in living cells using two cell lines, HT-29 cells and MDAMB-231 cells. Cells $\left(\sim 7 \times 10^{6}\right)$ were centrifuged to obtain cell pellets for imaging with a multispectral optoacoustic tomography (MSOT) imaging system (Fig. 3a). As expected, HT-29 cells incubated with the Rhodol-PA probe displayed a PA signal at $680 \mathrm{~nm}$ (Fig. 3b). When the cells were pretreated with the hNQO1 inhibitor dicoumarol followed by incubation with the probe, a lower PA signal was observed. Moreover, MDA-MB-231 cells incubated with the probe showed negligible PA response. These results were consistent with the literature reports that HT-29 cells had over-expressed hNQO1 levels and MDA-MB-231 cells had very low hNQO1 activity. ${ }^{27,28}$ This result suggested that the Rhodol-PA probe afforded an activatable molecular tool for specific differentiation and detection of hNQO1 activity in living cells using MSOT imaging.

Confocal laser scanning microscopy (CLSM) images revealed that HT-29 cells treated with Rhodol-PA displayed bright fluorescence in the cytosol (Fig. 3c and S16 $\dagger$ ). Further analysis showed that the fluorescence signal was not co-localized with mitochondria and lysosomes (Fig. S17†). Attenuated fluorescence was observed in HT-29 cells pretreated with the hNQO1 inhibitor followed by incubation with the probe (Fig. 3c and
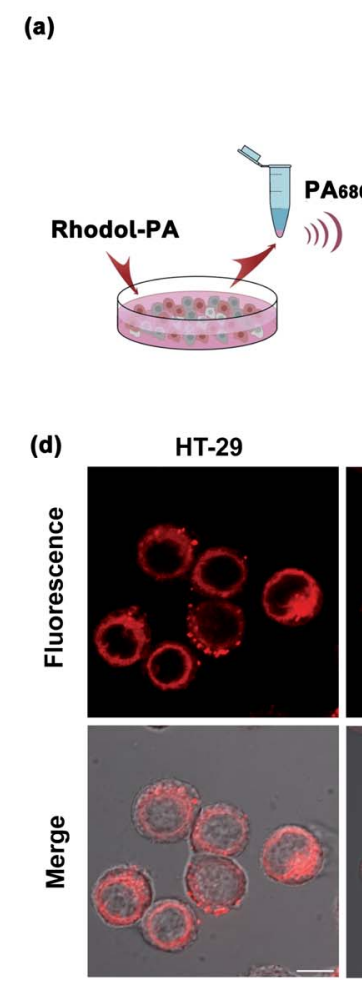

(b)

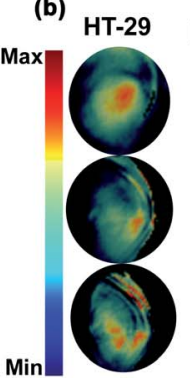

HT-29 + Dicoumarol

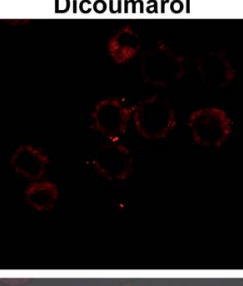

HT-29 +

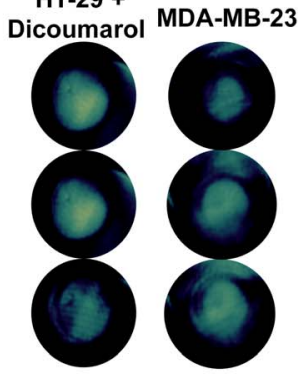

MDA-MB-231

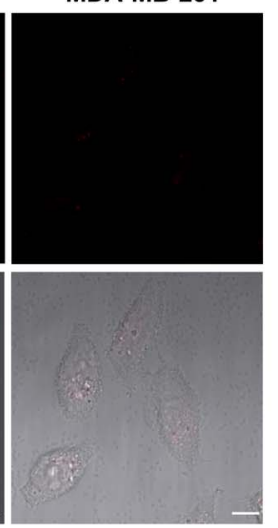

(c)

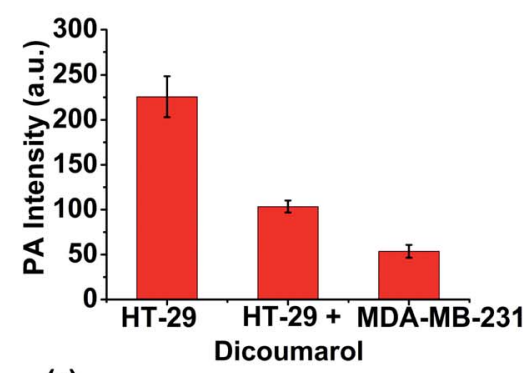

(e)

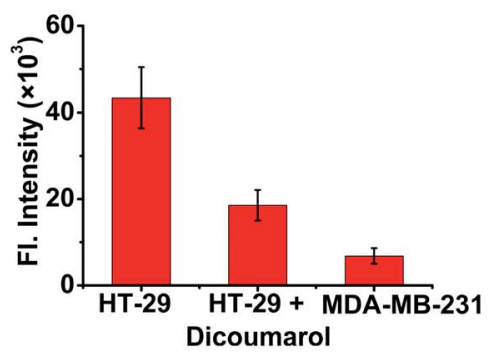

Fig. 3 PA and fluorescence imaging of $\mathrm{hNQO1}$ activity in living cells. (a) Schematic illustration of cells treated with Rhodol-PA followed by concentration for PA imaging. (b) Left and Middle: PA images of HT-29 cells incubated with Rhodol-PA (30 $\mu$ M) and HT-29 cells pretreated with the inhibitor $(100 \mu \mathrm{M})$ followed by incubation with Rhodol-PA (30 $\mu \mathrm{M})$. Right: PA images of MDA-MB-231 cells incubated with Rhodol-PA (30 $\mu$ M). (c) PA signal intensity at $680 \mathrm{~nm}$ for cells in (b). (d) Left and Middle: Confocal fluorescence images of HT-29 cells upon incubation with Rhodol-PA $(20 \mu \mathrm{M})$ in the absence or presence of the inhibitor $(100 \mu \mathrm{M})$. Right: Confocal images of MDA-MB-231 cells upon incubation with Rhodol-PA (20 $\mu$ M). Scale bar $=20 \mu \mathrm{m}$. (e) ROI analysis of fluorescence intensity in (d). 
$\mathrm{S} 16 \dagger)$. The fluorescence images were also very dim for MDA-MB231 cells incubated with the probe. These results demonstrated that the Rhodol-PA probe also enabled activatable fluorescence imaging of hNQO1, providing a PA/NIRF dual-mode platform for hNQO1 detection in living cells.

In addition, a methyl thiazolyltetrazolium (MTT) assay revealed that a concentration over $300 \mu \mathrm{M}$ of the Rhodol-PA probe had little effect on the cell viability (>90\%) of two cell lines, HT-29 and MDA-MB-231 (Fig. S18†), suggesting the potential of using this low-cytotoxicity Rhodol-PA probe for biological applications.

\section{PA/NIRF imaging of hNQO1 in tumor models}

Having demonstrated its ability to detect and image hNQO1 activity in living cells, we inspected the utility of the Rhodol-PA probe for imaging hNQO1 activity in living animals in dual PA/ NIRF mode. Tumor xenografts were generated via subcutaneous implantation of either HT-29 or MDA-MB-231 cells into the right oxter of BALB/c mice. The tumors were grown to specified sizes before imaging. As an initial study of the feasibility of the Rhodol-PA probe for in vivo visualization of hNQO1 activity in tumors, MSOT images were acquired at different intervals following intratumoral injection of Rhodol-PA. Obvious PA signals were obtained in $30 \mathrm{~min}$ post-injection for mice bearing HT-29 tumors, and the signals increased and reached a maximum at $\sim 3 \mathrm{~h}$ (Fig. $4 \mathrm{a}$ ). In contrast, there were negligible PA signals for mice bearing MDA-MB-231 tumors throughout the experiment. The ratio of the average PA increment for the HT-29 tumors to that for the MDA-MB-231 tumors was $\sim 8$-fold,

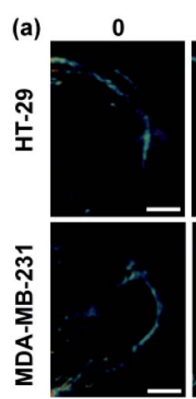

(b)

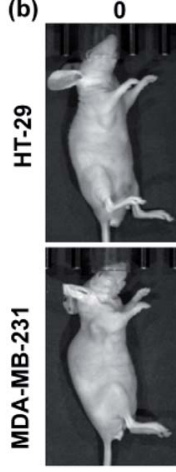

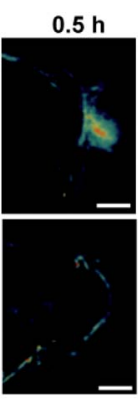

$0.5 \mathrm{~h}$

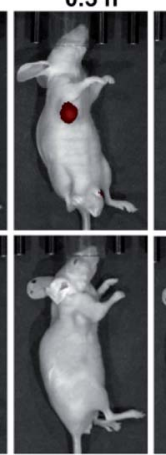

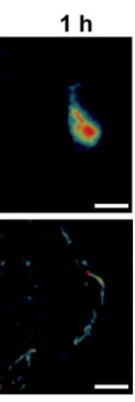

$1 \mathrm{~h}$

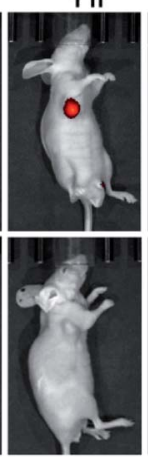

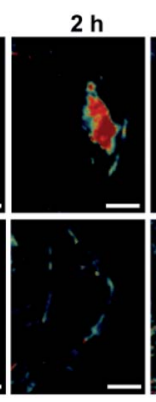

$2 \mathrm{~h}$

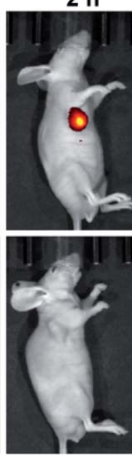

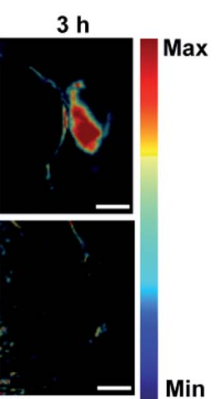

$3 \mathrm{~h}$

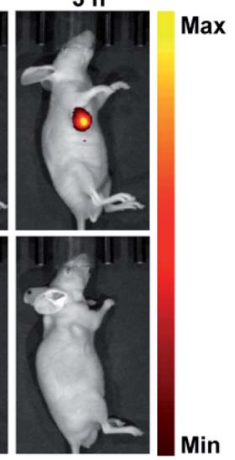

Fig. 4 Representative PA (a) and fluorescence (b) images of HT-29 and MDA-MB-231 tumor-bearing living mice at different time points upon intratumoral injection of Rhodol-PA (60 $\mu \mathrm{M}$ in $50 \mu \mathrm{L}$ PBS). Scale bar $=2 \mathrm{~mm}$ indicating the capability of the Rhodol-PA probe for highcontrast PA imaging of hNQO1-overexpressed tumors. Similar results were also obtained for fluorescence imaging studies (Fig. 4b). These results demonstrated the potential of the Rhodol-PA probe for rapidly and specifically detecting hNQO1 up-regulated tumors in living animals in dual PA/NIRF mode.

The ability of the Rhodol-PA probe to detect hNQO1 activity in living animals in dual PA/NIRF mode was then demonstrated. The Rhodol-PA probe or the PBS control was administered via tail injection to HT-29 and MDA-MB-231 tumor bearing mice, respectively. The MSOT and fluorescence images were acquired at different intervals $(0,0.5,1,3,5,7$, and $12 \mathrm{~h})$ following injection. We observed substantial PA signals at $1 \mathrm{~h}$ post-injection of the probe and the strongest signal was obtained $5 \mathrm{~h}$ after injection into tumor areas of the HT-29 cell xenografted mice (Fig. 5a and a1). The PA signals after injection of the PBS control did not show an appreciable increase throughout the experiment, demonstrating that the activation of PA signals arose from the reaction of the probe in the tumor areas (Fig. S19†). No appreciable increase in PA signals was found in tumor areas of the MDA-MB-231 cell xenografted mice after injection of the probe (Fig. 5a and a1). Fluorescence images exhibited similar responses in the tumor areas for the HT-29 and MDA-MB-231 cell xenografted mice (Fig. 5b and b1 and S20 $\dagger$ ). The data collected from six HT-29 tumor bearing mice in repetitive assays revealed that the average PA signals in the tumor areas exhibited a rapid increase in $1 \mathrm{~h}$ post-injection and reached the maximum at $5 \mathrm{~h}$ followed by a gradual decrease up to $12 \mathrm{~h}$ (Fig. 5a and a2). In contrast, the MDA-MB-231 tumor bearing mice displayed merely a slight increase of the PA signals in the tumor areas after injection. The ratio of average PA signals for the HT-29 tumors to that for the MDA-MB-231 tumors was $\sim 7$-fold, confirming that high-contrast PA imaging of hNQO1-overexpressed tumors was achieved using the Rhodol-PA probe. The data from NIRF images obtained with these tumor bearing mice also gave a consistent result; the HT29 tumors exhibited rapid fluorescence activation in $1 \mathrm{~h}$ while MDA-MB-231 tumors delivered almost negligible fluorescence responses (Fig. 5b and b2). Moreover, we found that in vivo PA spectra of the tumor areas in mice bearing HT-29 tumors obtained $5 \mathrm{~h}$ post-injection displayed a maximal PA signal at $680 \mathrm{~nm}$ with a spectral profile similar to the in vitro spectrum (Fig. 5a and a3). This result further verified that the PA signals in the HT-29 tumors were attributed to the specific activation of the Rhodol-PA probe mediated by hNQO1 overexpressed in HT29 cells. The in vivo PA spectra from the MDA-MB-231 tumor bearing mice also exhibited a similar but minor profile, indicating the low expression of hNQO1 in these cells.

In addition, ex vivo NIRF imaging of dissected organs including the heart, liver, spleen, lungs and kidneys at different times ( $5 \mathrm{~h}, 12 \mathrm{~h}$, and $24 \mathrm{~h}$ ) post-injection revealed that fluorescence signals dominantly appeared in the liver and kidneys (Fig. S21 $\dagger$ ). This finding indicated that the enzymatic product of Rhodol-PA was mainly metabolized and cleared in these two organs. Besides, fluorescence signals of the excised organs $24 \mathrm{~h}$ post injection were similar to those without administration of the probe, implying fast clearance of the metabolic products. 
(a)
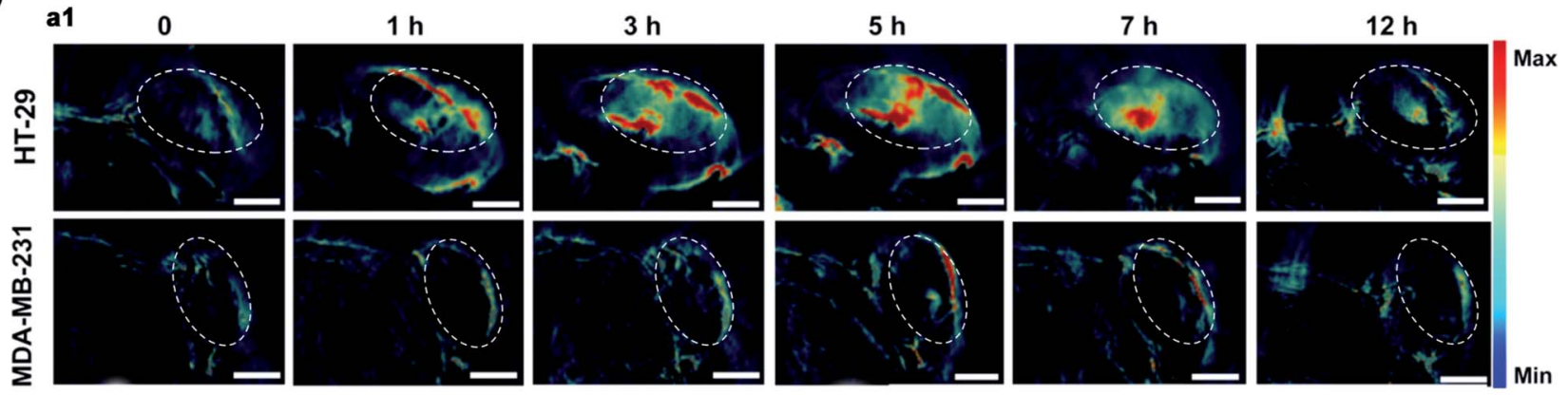

a2

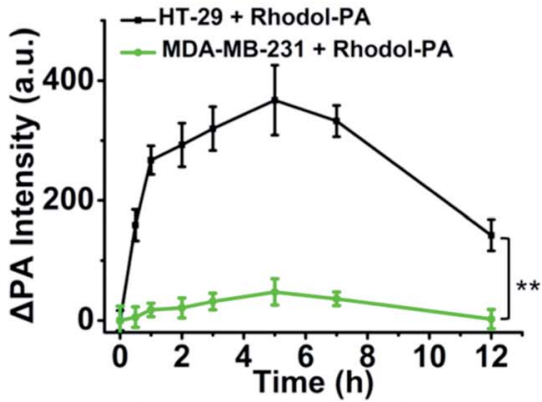

a3

(b)
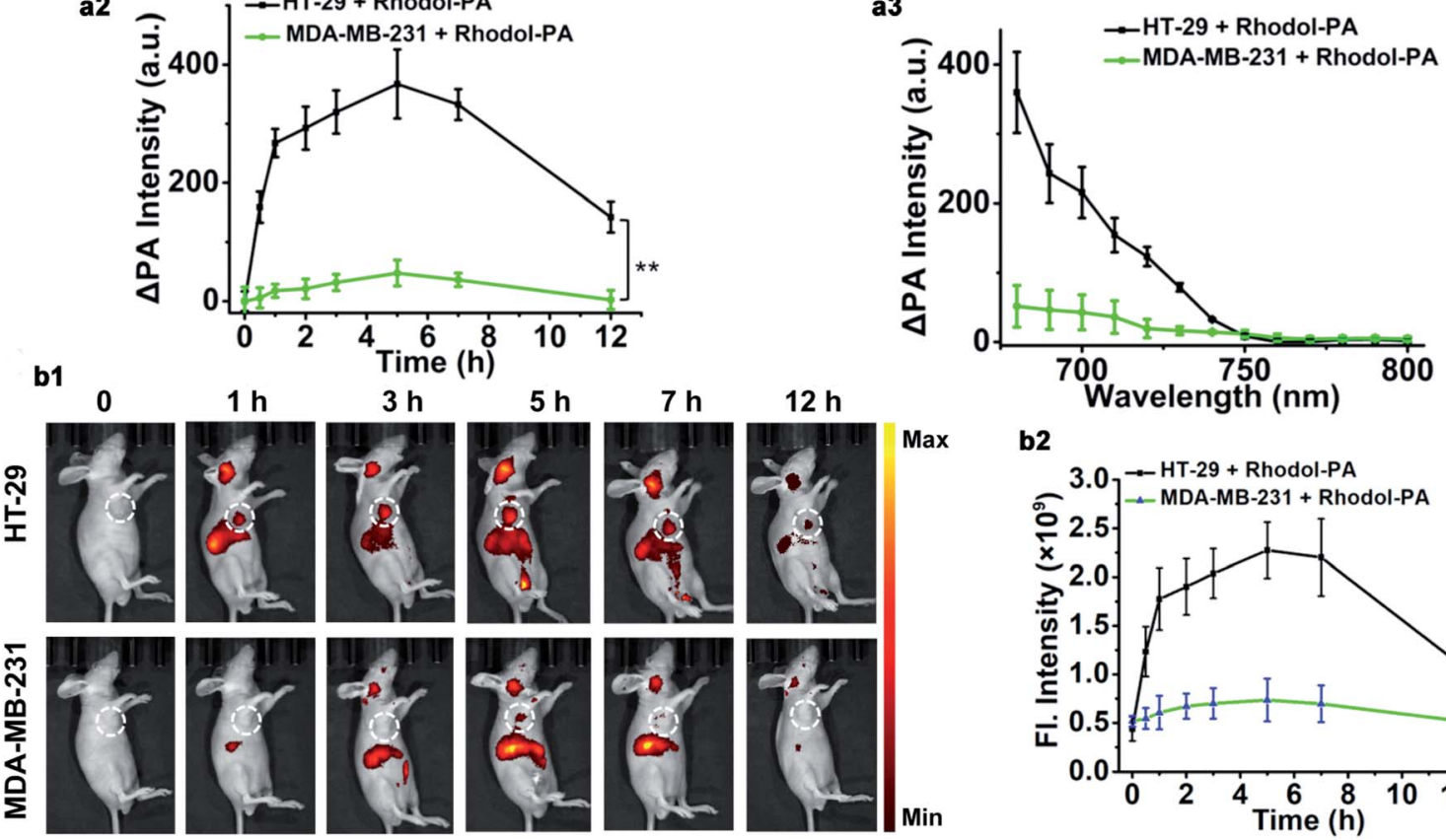

b2

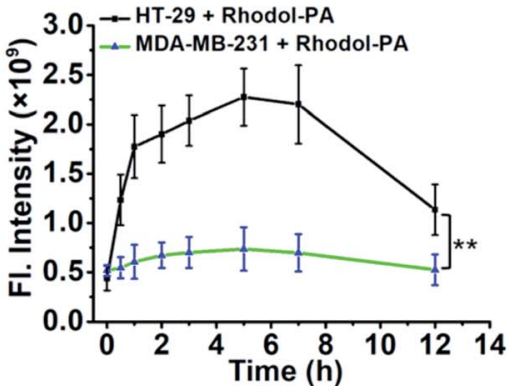

Fig. 5 In vivo PA and NIRF imaging of tumor hNQO1 activity. (a) PA imaging upon tail vein injection of Rhodol-PA (300 $\mu M$ in $200 \mu \mathrm{L} P B S$ ). (a1) Representative PA images of HT-29 and MDA-MB-231 tumor-bearing mice at different time points. Dotted lines indicated the tumor area. Scale bars $=2 \mathrm{~mm}$. (a2) Time-dependent PA variations of HT-29 and MDA-MB-231 tumor-bearing mice $(n=6)$. (a3) In vivo PA spectra from both HT29 and MDA-MB-231 tumor-bearing mice $5 \mathrm{~h}$ post injection. (b) In vivo fluorescence imaging upon tail vein injection of Rhodol-PA (500 $\mu \mathrm{M}$ in $200 \mu$ L PBS). (b1) Representative fluorescence images of HT-29 and MDA-MB-231 tumor-bearing mice at different time points. Dotted lines indicated the tumor area. (b2) Time-dependent fluorescence variations of HT-29 and MDA-MB-231 tumor-bearing living mice $(n=6)$. Statistical significance represents a comparison of mice bearing HT-29 tumors to mice bearing MDA-MB-231 tumors. Error bars represent SD. $* * p<0.01$.

Altogether, the live mouse imaging results demonstrated that our probe could be selectively activated in hNQO1 up-regulated tumors in living animals, enabling PA/NIRF dual-mode imaging of tumors with over-expression of hNQO1, implying its potential for in vivo tumor diagnostics and treatment evaluation.

\section{Conclusions}

We developed a high-contrast activatable PA probe based on the analyte-induced spirocyclic ring-opening strategy of spirocyclic xanthene dyes. A new Rhodol-NIR chromophore with a large extinction coefficient, superb photostability, low quantum yield and spirocyclic ring-opening activation was engineered. This new dye was used to develop an activatable PA probe, allowing high-contrast PA/NIRF dual-mode detection and imaging of hNQO1. The Rhodol-PA probe was demonstrated to exhibit high sensitivity, high selectivity and high-signal-to-background detection of hNQO1 activity in vitro via $\mathrm{PA}$, absorption and fluorescence measurements. It was also successfully utilized to image and differentiate hNQO1 activity with high contrast in living cells and tumor bearing animals in PA/NIRF dual-mode imaging. To our knowledge, this is the first time that a rhodol derivative has been developed with NIR absorption, low quantum yield and generally applicable responsiveness. This unique spirocyclic ring-opening strategy could provide a new paradigm for developing high-contrast PA probes for in vivo biomedical imaging applications.

\section{Conflicts of interest}

There are no conflicts to declare. 


\section{Acknowledgements}

This work was supported by the National Natural Science Foundation of China (21527810, 91753107, and 21521063).

\section{Notes and references}

1 L. V. Wang and J. Yao, Nat. Methods, 2016, 13, 627-638.

2 A. P. Jathoul, J. Laufer, O. Ogunlade, B. Treeby, B. Cox, E. Zhang, P. Johnson, A. R. Pizzey, B. Philip, T. Marafioti, M. F. Lythgoe, R. B. Pedley, M. A. Pule and P. Beard, Nat. Photonics, 2015, 9, 239-246.

3 A. Taruttis and V. Ntziachristos, Nat. Photonics, 2015, 9, 219227.

4 S. Zackrisson, S. M. W. Y. Van De Ven and S. S. Gambhir, Cancer Res., 2014, 74, 979-1004.

5 L. V. Wang and S. Hu, Science, 2012, 335, 1458-1462.

6 J. Weber, P. C. Beard and S. E. Bohndiek, Nat. Methods, 2016, 13, 639-650.

7 K. Y. Pu, A. J. Shuhendler, J. V. Jokerst, J. G. Mei, S. S. Gambhir, Z. N. Bao and J. H. Rao, Nat. Nanotechnol., 2014, 9, 233-239.

8 W. Gao, Y. H. Sun, M. Cai, Y. J. Zhao, W. H. Cao, Z. H. Liu, G. W. Cu and B. Tang, Nat. Commun., 2018, 9, 231.

9 A. A. Dragulescu, S. R. Kothapalli, G. A. Tikhomirov, J. H. Rao and S. S. Gambhir, J. Am. Chem. Soc., 2013, 135, 1101511022.

10 Q. Q. Miao and K. Y. Pu, Bioconjugate Chem., 2016, 27, 28082823.

11 H. Li, P. Zhang, L. P. Smaga, R. A. Hoffman and J. Chan, J. Am. Chem. Soc., 2015, 137, 15628-15631.

12 S. Roberts, M. Seeger, Y. Y. Jiang, A. Mishra, F. Sigmund, A. Stelzl, A. Lauri, P. Symvoulidis, H. Rolbieski, M. Preller, X. L. D. Ben, D. Razansky, T. Orschmann, S. C. Desbordes, P. Vetschera, T. Bach, V. Ntziachristos and G. G. Westmeyer, J. Am. Chem. Soc., 2018, 140, 2718-2721.

13 J. J. Zhang, X. Zhen, P. K. Upputuri, M. Pramanik, P. Chen and K. Y. Pu, Adv. Mater., 2017, 29, 1604764.

14 C. J. Reinhardt, E. Y. Zhou, M. D. Jorgensen, G. Partipilo and J. Chan, J. Am. Chem. Soc., 2018, 140, 1011-1018.

15 H. J. Knox, J. Hedhli, T. W. Kim, K. Khalili, L. W. Dobrucki and J. Chan, Nat. Commun., 2017, 8, 1794.
16 X. Zhen, J. J. Zhang, J. G. Huang, C. Xie, Q. Q. Miao and K. Y. Pu, Angew. Chem., Int. Ed., 2018, 57, 7804-7808.

17 J. Zhang, L. P. Smaga, N. S. R. Satyavolu, J. Chan and Y. Lu, J. Am. Chem. Soc., 2017, 139, 17225-17228.

18 J. Levi, S. R. Kothapalli, T. J. Ma, K. Hartman, B. T. KhuriYakub and S. S. Gambhir, J. Am. Chem. Soc., 2010, 132, 11264-11269.

19 X. Y. Bai, Y. Y. Huang, M. Y. Lu and D. Yang, Angew. Chem., Int. Ed., 2017, 56, 1-6.

20 B. C. Dickinson and C. J. Chang, J. Am. Chem. Soc., 2008, 130, 9638-9639.

21 R. S. Kathayat, P. D. Elvira and B. C. Dickinson, Nat. Chem. Biol., 2017, 13, 150.

22 S. Bhuniya, S. Maiti, E. J. Kim, H. Lee, J. L. Sessler, K. S. Hong and J. S. Kim, Angew. Chem., Int. Ed., 2014, 53, 4469-4474.

23 T. D. Horvath, G. Kim, R. Kopelman and S. Ashkenazi, Analyst, 2008, 133, 747-749.

24 J. G. Jo, C. H. Lee, R. Kopelman and X. D. Wang, Nat. Commun., 2017, 8, 471.

25 T. Ikeno, K. Hanaoka, S. Iwaki, T. Myochin, Y. Murayama, H. Ohde, T. Komatsu, T. Ueno, T. Nagano and Y. Urano, Anal. Chem., 2019, 91, 9086-9092.

26 X. Q. Chen, T. Pradhan, F. Wang, J. S. Kim and J. Yoon, Chem. Rev., 2012, 112, 1910-1956.

27 W. C. Silvers, B. Prasai, D. H. Burk, M. L. Brown and R. L. McCarley, J. Am. Chem. Soc., 2013, 135, 309-314.

28 N. Kwon, M. K. Cho, S. J. Park, D. Kim, S. J. Nam, L. Cui, H. M. Kim and J. Yoon, Chem. Commun., 2017, 53, 525-528.

29 S. U. Hettiarachchi, B. Prasai and R. L. McCarley, J. Am. Chem. Soc., 2014, 136, 7575-7578.

30 J. Chan, S. C. Dodani and C. J. Chang, Nat. Chem., 2012, 4, 973-984.

31 J. B. Grimm, A. K. Muthusamy, Y. J. Liang, T. A. Brown, W. C. Lemon, R. Patel, R. W. Lu, J. J. Macklin, P. J. Keller, N. Ji and L. D. Lavis, Nat. Methods, 2017, 14, 987-994.

32 L. Yuan, W. Y. Lin and H. Chen, Biomaterials, 2013, 34, 95669571.

33 H. S. Jung, J. H. Lee, K. Kim, S. Y. Koo, P. Verwilst, J. L. Sessler, C. H. Kang and J. S. Kim, J. Am. Chem. Soc., 2017, 139, 9972-9978. 